\title{
Thermally excited fluctuations as a pure electron plasma temperature diagnostic
}

\author{
N. Shiga, ${ }^{a}$ F. Anderegg, D. H. E. Dubin, and C. F. Driscoll \\ Institute for Pure and Applied Physical Sciences and Department of Physics, \\ University of California at San Diego, La Jolla, California 92093 \\ R. W. Gould \\ California Institute of Technology, Mail Stop 128-95, Pasadena, California 91103
}

(Received 16 November 2005; accepted 18 January 2006; published online 16 February 2006)

\begin{abstract}
Thermally excited charge fluctuations in pure electron plasma columns provide a diagnostic for the plasma temperature over a range of $0.05<k_{\mathrm{B}} T<10 \mathrm{eV}$. Three different nonperturbative methods have been developed to determine the plasma temperature. The first method fits the near-Lorentzian spectrum of thermal fluctuations near a single weakly damped mode. This method works well where the modes are weakly damped, i.e., when $\lambda_{\mathrm{D}} / R_{p}<0.3$. The second method utilizes the emission spectrum over a broad frequency range encompassing several modes and the nonresonant fluctuations between modes. This method works for long columns with $\lambda_{\mathrm{D}} / R_{p}>0.2$, so that Landau damping is dominant and well modeled by theory. The third method compares the total (frequency-integrated) number $\delta N$ of fluctuating image charges on the wall antenna to a simple thermodynamic calculation. This method works when $\lambda_{\mathrm{D}} / R_{p}>0.2$. (C) 2006 American Institute of Physics. [DOI: 10.1063/1.2172928]
\end{abstract}

\section{INTRODUCTION}

Penning-Malmberg traps provide the long-time confinement of single-species plasma columns with static electric and magnetic fields. These traps are widely used to confine $N=10^{3}-10^{9}$ electrons, ions, or antimatter in plasma experiments, atomic physics, ${ }^{1}$ and spectroscopy. ${ }^{2}$ The confined plasmas typically relax to near-thermal-equilibrium states, characterized by a plasma temperature $T_{p}$, density $n$, plasma radius $R_{p}$, and plasma length $L_{p}$. Here, we describe techniques to obtain $T_{p}$ (and other parameters) from the passive reception of image charge fluctuations on wall cylinders.

The thermal equilibrium plasma in a Penning-Malmberg trap varies in a wide range of plasma regimes, from lasercooled Coulomb crystals ${ }^{3}$ to the gaseous regime that is reported here. The fluctuating current on wall cylinders is induced by the motion of the charged particles. Whether this particle motion is coherent or not depends on the plasma regime, characterized by Debye length $\lambda_{\mathrm{D}}$.

In the high-temperature limit, this fluctuation spectrum represents the random motion of electrons with no mutual interactions, as in an ideal gas. Therefore, the density fluctuation spectrum reflects the thermal velocity distribution of the plasma, which is a Gaussian with width $\sim v_{\text {th }} / L_{p}$. Preliminary work by the group at Brigham Young University ${ }^{4}$ focused on the fluctuation spectrum between modes in the ideal gas limit.

As the temperature decreases from this high-temperature limit, Debye shielding suppresses the random motion of particles, and the collective effects (i.e., waves) become dominant. These weakly damped plasma waves may be considered to be the normal modes of the system. In traps with a

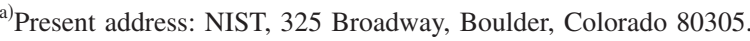

finite length and radius, these modes appear at discrete Trivelpiece-Gould (T-G) standing mode frequencies. ${ }^{5,6}$ In an isolated equilibrium plasma, each standing mode would have an average electrostatic potential energy of $1 / 2 k_{\mathrm{B}} T$ per mode. In practice, the mode coupling to the receiver electronics at "temperature" $T_{\ell}$ can be comparable to the coupling to the plasma, complicating the picture slightly. For simplicity, we generally express temperatures in energy units, $T_{p}(\mathrm{eV}) \equiv k_{\mathrm{B}} T$.

Historically, the (somewhat simpler) center-of-mass "trap modes" have been observed with tuned resonant circuits, ${ }^{7}$ to diagnose the number of particles, but not their temperature. At higher frequencies, the thermal excitation of cyclotron modes are readily observed in warm non-neutral ${ }^{8}$ and hot fusion plasmas; ${ }^{9}$ upper hybrid modes have also been used as a thermal diagnostic. ${ }^{10}$ Temperature information can also be obtained by measuring changes in the frequencies of particular modes; this is in some sense a simpler version of the methods developed here. ${ }^{11}$ In space plasmas, thermal noise diagnostics ${ }^{12}$ are substantially different because of the lack of boundaries. In the crystallized regime, the equipartition of mode energy has been observed in dusty plasmas. ${ }^{13} \mathrm{In}$ the regime of macroscopic solids, thermally excited vibrations of laser resonator mirrors ${ }^{14}$ are carefully analyzed, assuming that each mode has energy $T$.

The ionospheric microwave backscattering observations in the 1960s were the first observations of the fluctuation spectrum in plasmas. ${ }^{15}$ The scattering is from electrons that are "bound" to ions, and therefore the spectrum of the backscattering around the incident wave frequency reflects the ion motion, instead of the electron motion. The observed spectrum represented a strongly damped ion-acoustic wave on top of a Gaussian ion velocity distribution. ${ }^{16}$ Theory calculations predicted the transition of ion-acoustic waves from 
strongly damped to weakly damped as $T_{e} / T_{i}$ increases, but no ionospheric temperature control was possible. Here, the analogous transition is observed as we change the temperature of the electron plasma.

In this paper, we present observations of thermally excited fluctuations in pure electron plasmas over a temperature range of $0.05<T_{p}<10 \mathrm{eV}$, using a room temperature $(0.03 \mathrm{eV})$ receiver. At high temperature $\left(\lambda_{\mathrm{D}} / R_{p}>0.3\right)$, the spectrum is a broad Gaussian that reflects velocity distribution of the electrons, with heavily damped modes visible, in addition. As the temperature decreases, the broad Gaussian spectrum decreases in amplitude due to Debye shielding; and, instead, the mode peaks rise up because Landau damping decreases exponentially as the temperature decreases.

We have developed three different nonpertubative strategies to determine the plasma temperature from passive reception of cylindrically symmetric $\left(m_{\theta}=0\right)$ image charge fluctuations. The first method utilizes fits to a single weakly damped Trivelpiece-Gould mode, typically the lowest mode with $k_{z}=1$. Near the isolated mode, the plasma/antenna admittance $Z_{p}^{-1}$ is well modeled as a simple pole, with parameters for amplitude, frequency, and damping. Nyquist's theorem then relates the mode amplitude squared to $T_{p}$ times an antenna coupling $\mathcal{G}$, with modifications arising from the receiver (load) impedance $Z_{\ell}$. In practice, temperatures $T_{p}$ accurate to $\pm 20 \%$ can be obtained from a FFT analysis of about $0.01 \mathrm{~s}$ of $\mathrm{rf}$ data, or from slow spectrum analyzer (tuned filter) scans over tens of seconds.

The second method utilizes a broad received spectrum spanning several T-G modes and the intervening nonresonant fluctuations. Fitting this spectrum to the predictions of kinetic theory (for a uniform plasma) gives $T_{p}$, and also gives $n, R_{p}$, and $L_{p}$.

In some plasma regimes, this gives a stunningly accurate fit to multiple modes. In practical terms, this requires careful calibration of receiver sensitivity versus frequency, including the effects of cable resonances. More fundamentally, the technique fails for short columns because the theory with periodic boundary conditions does not accurately model Landau damping of finite-length modes.

The third technique compares the total (frequencyintegrated) fluctuations to the predictions of thermodynamics. The measured fluctuations are proportional to $T_{p}$ for $\lambda_{\mathrm{D}} / R_{p} \lesssim 1$, as expected; and are predicted to saturate in the ideal gas limit of $\lambda_{\mathrm{D}} / R_{p}>1$. For an idealized receiver with uniform gain versus frequency, this measurement corresponds to a simple time average of the received image charge voltage squared. In practice, the received voltage must be Fourier analyzed and frequency weighted before being frequency integrated. Nevertheless, the technique provides a fundamental and practical connection to the thermodynamics of these simple many-body systems.

\section{APPARATUSES AND MODES}

Fluctuation measurements were obtained from pure electron plasmas contained in two similar Penning-Malmberg traps ("IV" and "EV") shown schematically in Fig. 1. These two traps differ mainly in plasma diameter and magnetic

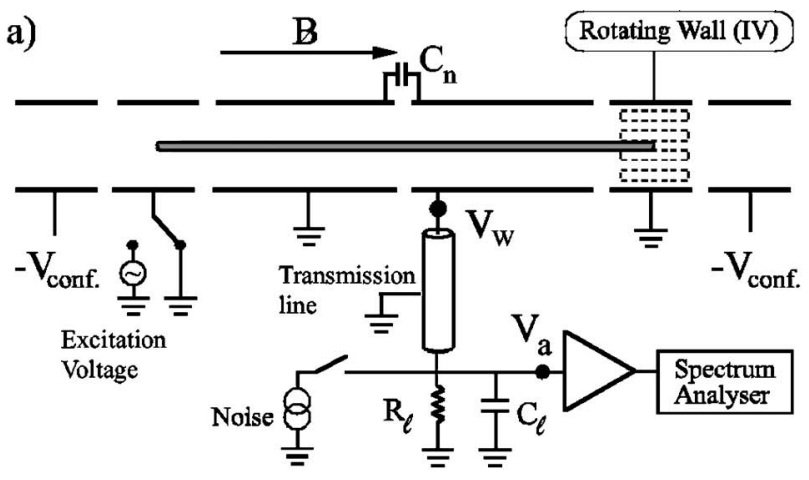

b)

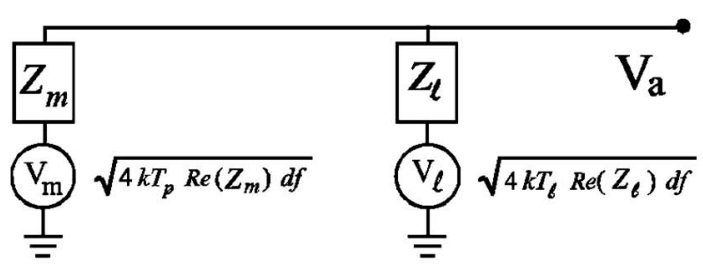

c)

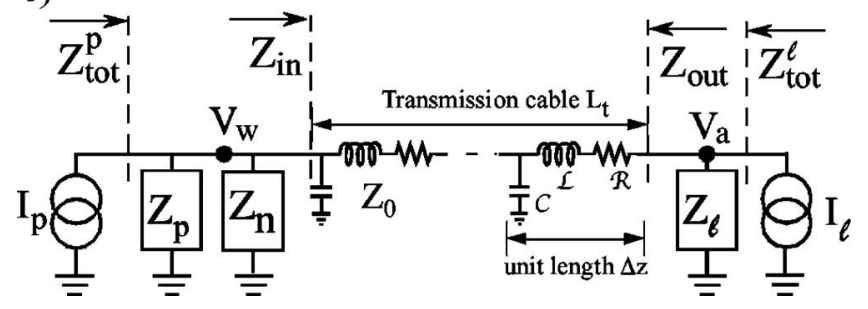

FIG. 1. (a) Schematic diagram of plasma emission measurement. (b) Circuit analog to the plasma mode and antenna. (c) Circuit analog of plasma, load, coupling to neighboring cylinder and transmission cable.

field strength. The IV trap consists of a series of hollow conducting cylinders of radius $R_{w}=2.86 \mathrm{~cm}$ contained in an ultrahigh vacuum at $P \approx 10^{-10}$ Torr with a uniform axial magnetic field of $B_{z}=30 \mathrm{kG}$. Electrons are injected from a hot tungsten filament, and contained axially by voltages $V_{\text {conf }} \approx-200 \mathrm{~V}$ on end electrodes. Typical plasmas have $N$ $\approx 10^{9}$ electrons in a column length $L_{p} \approx 41 \mathrm{~cm}$, with a plasma radius $R_{p} \approx 0.2 \mathrm{~cm}$ and a central density $n_{0}$ $\approx 10^{7} \mathrm{~cm}^{-3}$. (For EV, the parameters are $B_{z}=0.375 \mathrm{kG}, R_{p}$ $=1.7 \mathrm{~cm}, R_{w}=3.8 \mathrm{~cm}$, and $L_{p}=15-37 \mathrm{~cm}$.)

The plasma density profile $n(r)$ and the temperature $T_{p}$ are obtained by dumping the plasma axially and measuring the total charge passing through a hole in a scanning collimator plate. Both measurements require shot-to-shot reproducibility of the injected plasma, and we typically observe variability $\delta n / n \leqslant 1 \%$. On IV, a weak "rotating wall" drive at $f_{\mathrm{RW}} \sim 0.5 \mathrm{MHz}$ is used to obtain steady-state confinement of the electron column. ${ }^{17,18}$ On EV, we did not apply a rotating wall drive, so the plasmas expand radially toward the wall with a characteristic "mobility" time of $\tau_{m} \approx 100 \mathrm{~s}$; measurements are taken on the electron plasmas, which are repetitively injected and dumped. The analog spectrum analyzer scans reported here typically required about $0.5 \mathrm{~s}$ to complete; whereas the FFT analysis used transient digitizer scans of only $10 \mathrm{~ms}$ duration.

To control the plasma temperature, we apply auxiliary 


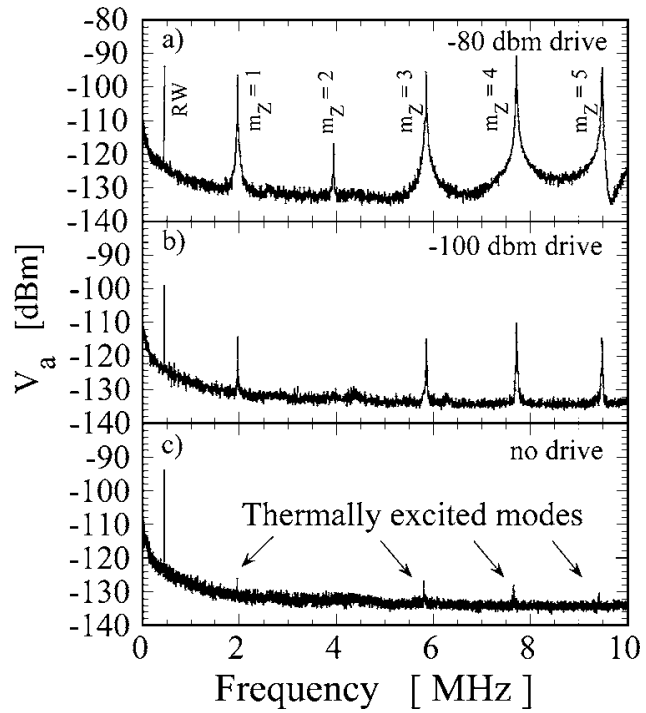

FIG. 2. Spectrum of $m_{r}=1, m_{\theta}=0, m_{z}=1,2, \ldots, 5$ Trivelpiece-Gould modes for three drive amplitudes including no drive, i.e., thermally excited.

"wiggle" heating by modulating one electrode voltage at a frequency $f_{h}=0.8-1.0 \mathrm{MHz}$, where $f_{h}$ is chosen so that all harmonics are distinct from the T-G mode of interest. On the EV apparatus, a "heating burst" is applied before the measurement; on the IV apparatus, the heating is applied continuously to balance the cooling due to cyclotron radiation.

Our new passive-reception temperature diagnostic techniques are compared against traditional destructive temperature diagnostics that give the parallel and perpendicular temperatures $T_{\|}$and $T_{\perp}(r)$. The $T_{\perp}$ diagnostic on the EV apparatus operates by measuring the change in the parallel energy of the dumped electron when a secondary magnetic field causes some perpendicular energy to be transformed to the parallel direction. This is commonly called a "magnetic beach" analyzer. ${ }^{19}$

In IV, the (central) parallel temperature $T_{\|}(r=0)$ is measured using an "evaporative" technique. ${ }^{20}$ For this measurement, the dump voltage is slowly ramped to ground ( $\sim 10 \mathrm{~ms})$, and the number of escaping electrons versus ramped voltage is digitized and fit to the tail of a Maxwellian distribution, which defines $T_{\|}$. In practice, we take the average value of three shots, and the shot-to-shot deviation is typically $\delta T_{\|} / T_{\|} \approx 0.2$.

We analyze long-wavelength, $\theta$-symmetric electron plasma modes, because these modes give the largest image charge signals on the wall antenna. The cylindrical bounding wall causes these modes to have an "acoustic" spectrum, with $\omega \propto k_{z}$.

We performed the transmission experiment by applying sinusoidal voltage on the end cylinder and measuring the voltage on the receiving antenna [Fig. 1(a)] as we swept the frequency $f=0.01-10 \mathrm{MHz}$. This antenna is connected to an amplifier outside the vacuum chamber with a coaxial cable. Below $5 \mathrm{MHz}$, this coaxial cable can be approximated as an additional capacitance $C_{\ell}$. The input impedance of the amplifier is $690 \Omega$. The plasma fluctuation signal is amplified, then fed to the spectrum analyzer. Figures 2(a) and 2(b) show

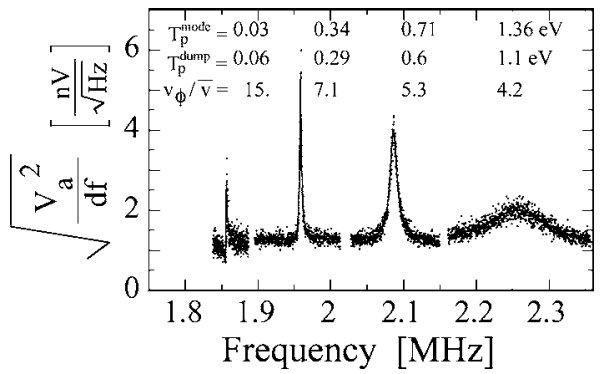

FIG. 3. Spectra of the thermally excited $m_{r}=1, m_{\theta}=0, m_{z}=1$, mode for different plasma temperatures; the solid lines are fits to Eq. (5). The temperature $T_{p}^{\text {mode }}$ is from emission measurements. The temperature $T_{p}^{\text {dump }}$ is from the standard dump technique.

the results at two different excitation amplitudes, showing the spectrum of azimuthally symmetric $m_{\theta}=0$ standing Trivelpiece-Gould modes.

The frequencies of these "Trivelpiece-Gould" mode resonances for $m_{\theta}=0$ and $m_{r}=1$ can be approximated in the limit of $k_{z} \lambda_{\mathrm{D}} \ll 1$ and $R_{p} k_{z} \ll 1$ as

$$
\omega_{m} \approx \omega_{p}\left(\frac{R_{p}}{R_{w}}\right)\left(r_{w} k_{z}\right)\left(\frac{1}{2} \ln \frac{R_{w}}{R_{p}}\right)^{1 / 2}\left[1+\frac{3}{2}\left(\frac{\bar{v}}{v_{\phi}}\right)^{2}\right],
$$

with damping

$$
\gamma \approx-\sqrt{\frac{\pi}{8}} \omega\left(\frac{v_{\phi}}{\bar{v}}\right)^{3} \exp \left[-\frac{1}{2}\left(\frac{v_{\phi}}{\bar{v}}\right)^{2}\right] .
$$

The wave frequencies scale with the plasma frequency $f_{p}$ $\equiv \omega_{p} / 2 \pi=28 \mathrm{MHz}\left(n / 10^{7} \mathrm{~cm}^{-3}\right)^{1 / 2}$, are reduced by the fill ratio $R_{p} / R_{w}$ and by the trap radius compared to the axial wavelength. $^{21}$ The modes are typically described theoretically in the "infinite length" limit, where they would be written as $\delta n \sim A \delta n(r) \exp \left(i k_{z} z-i \omega_{m} t-\gamma_{m} t\right)$. The axial wave numbers for resonant standing modes in length $L_{p}$ are given approximately by $k_{z}=\pi m_{z} / L_{p}$, where the axial mode number is $m_{z}=1,2 \ldots$. The thermal corrections depend on the ratio of thermal velocity $v_{\text {th }} \equiv(T / m)^{1 / 2}$ to the wave phase velocity $v_{\phi}$, where $v_{\phi} \approx \omega_{m} / k_{z}$ is ill defined to the extent that $k_{z}$ is approximate.

\section{SINGLE MODE ANALYSIS (REF. 22)}

Figure 2(c) shows that small peaks representing thermally excited modes are still above the noise level, even when the transmitting electrode is grounded.

Figure 3 shows the received spectra of thermally excited $m_{z}=1$ modes for four different plasma temperatures from 0.03 to $1.4 \mathrm{eV}$. The spectra are nominally Lorentzian for these weakly damped modes. The mode frequency $f_{m}$ increases by $20 \%$ with the elevated temperature, as expected from Eq. (1). The mode damping $\gamma_{m}$ also increases exponentially with the temperature increases, as expected from Eq. (2).

This fluctuation is the Johnson noise generated across plasma-antenna impedance, $Z_{p}(\omega)$, illustrated in Fig. 1(b). The Nyquist theorem predicts that the fluctuating current is proportional to the temperature and to the dissipative (real) part of the admittance, $Z_{p}^{-1}$. We attach a load $Z_{\ell}$ (that has its 
own fluctuating current source) to obtain a signal, as shown in Fig. 1(b). The overall voltage measured at the load is therefore the sum of the fluctuating currents, inducing a voltage on the total impedance of $Z_{p}$ and $Z_{\ell}$ in parallel. This gives

$$
\begin{aligned}
\frac{V_{a}^{2}}{d f} & =\left(\frac{I_{p}^{2}}{d f}+\frac{I_{\ell}^{2}}{d f}\right)\left|\frac{Z_{p} Z_{\ell}}{Z_{p}+Z_{\ell}}\right|^{2} \\
& =\left(4 T_{p} \operatorname{Re}\left\{Z_{p}^{-1}\right\}+4 T_{\ell} \operatorname{Re}\left\{Z_{\ell}^{-1}\right\}\right)\left|\frac{Z_{p} Z_{\ell}}{Z_{p}+Z_{\ell}}\right|^{2} .
\end{aligned}
$$

Here $I_{p}$ and $I_{\ell}$ are added in quadrature since they are not correlated. The determination of $Z_{p}(\omega)$ is the key to determine $T_{p}$ from $V_{a}^{2} / d f$, once we have determined $I_{\ell}^{2} / d f$ and $Z_{\ell}(\omega)$ from separate measurements.

When $T_{p}$ is small such that $\lambda_{\mathrm{D}} / R_{p}<0.3$, the low-lying modes are weakly damped and dominant, with frequency $\omega_{m}=2 \pi f_{m}$ and damping $\gamma_{m}$. Near this weakly damped mode, the plasma admittance $Z_{p}^{-1}$ is approximated by the singlemode admittance $Z_{m}^{-1}$, which is the simple pole,

$$
Z_{p}^{1}(\omega) \approx Z_{m}^{1}(\omega) \equiv \frac{\mathcal{G} \omega_{m}^{2}}{i\left(\omega-\omega_{m}\right)+\gamma_{m}} .
$$

Here, $\mathcal{G}$ is a geometrical capacitive coupling coefficient, representing the capacitance between the wall antenna and the plasma. Using this simple pole $Z_{m}^{-1}$ in (3), we obtain the fluctuation spectrum as

$$
\begin{aligned}
\frac{V_{a}^{2}(f)}{d f}= & 4 T_{p} Z_{m}^{\mathrm{Re}}\left(\omega_{m}\right) \frac{\left|Z_{\ell}\right|^{2}}{\left|Z_{m}^{\mathrm{Re}}\left(\omega_{m}\right)+Z_{\ell}^{\mathrm{Re}}\right|^{2}} \frac{\gamma_{\mathrm{tot}}^{2}}{\gamma_{\mathrm{tot}}^{2}+\left(\omega-\omega_{m}^{\prime}\right)^{2}} \\
& +4 k T_{\ell} Z_{\ell}^{\mathrm{Re}}\left(1-\frac{2\left(\omega-\omega_{m}^{\prime}\right) \delta \omega_{m}+\left(\gamma_{\mathrm{tot}}^{2}-\gamma_{m}^{2}-\delta \omega_{m}^{2}\right)}{\gamma_{\mathrm{tot}}^{2}+\left(\omega-\omega_{m}^{\prime}\right)^{2}}\right),
\end{aligned}
$$

where

$$
\begin{aligned}
& \gamma_{\mathrm{tot}} \equiv \gamma_{m}+\gamma_{\ell} \equiv\left(1+\frac{Z_{\ell}^{\mathrm{Re}}}{Z_{m}^{\mathrm{Re}}\left(\omega_{m}\right)}\right) \gamma_{m}, \\
& \delta \omega_{m} \equiv \omega_{m}-\omega_{m}^{\prime} \equiv Z_{\ell}^{\mathrm{Im}} \omega_{m}^{2} \mathcal{G}, \\
& Z_{m}^{\mathrm{Re}}\left(\omega_{m}\right)=\gamma_{m} / \mathcal{G} \omega_{m}^{2},
\end{aligned}
$$

with $Z^{\operatorname{Re}} \equiv \operatorname{Re}\{Z\}$ and $Z^{\operatorname{Im}} \equiv \operatorname{Im}\{Z\}$.

There are five characteristics of the spectrum that determines five free parameters, $T_{p}, \omega_{m}, \gamma_{m}, T_{\ell}$, and $\mathcal{G}$ in Eq. (5). Three parameters are determined by the first term of Eq. (5) that gives a Lorentzian spectrum, which are mode frequency $\left(\omega_{m}\right)$, width $\left(\gamma_{m}\right)$, and area $\left(T_{p}\right)$. Here two more parameters are determined from the second term: the amplitude of the "white" noise level $\left(T_{\ell}\right)$, and the dip and peak $(\mathcal{G})$. In order to determine all five parameters from one emission spectrum, all five characteristics need to be significant.

Figure 4(a) shows a case that dip and peak are smaller than the scatter of the data. In this case, $\mathcal{G}$ needs to be determined by a separate reflection measurement. ${ }^{23}$ Figure 4(b) shows the case where all five parameters are determined from a single spectrum. The measurement condition is the

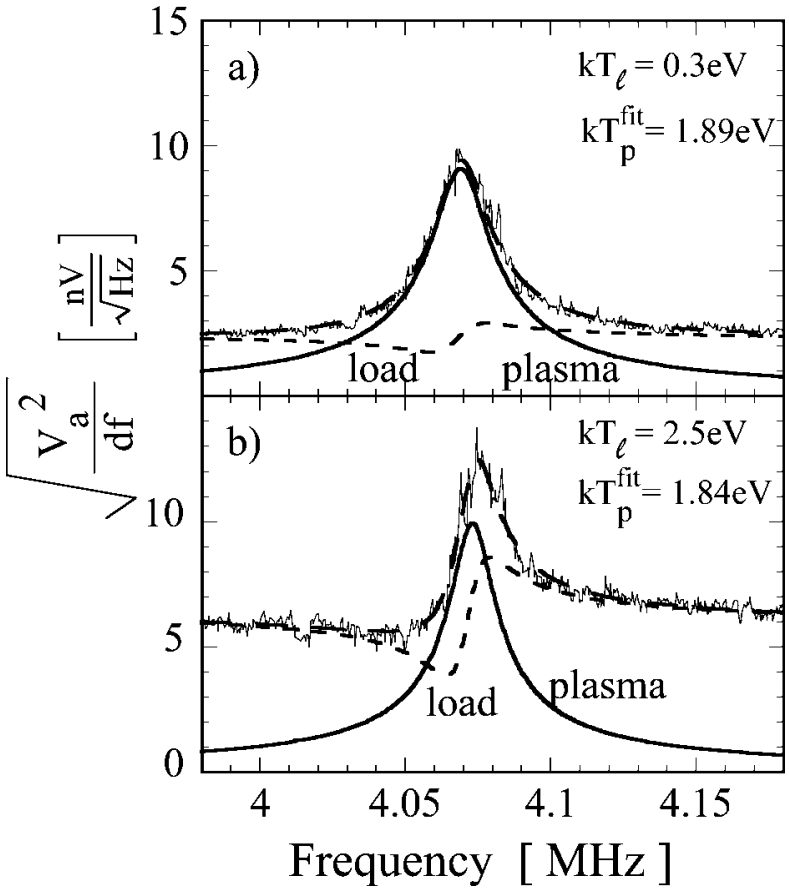

FIG. 4. (a) Spectra of the thermally excited $m_{\theta}=0, m_{z}=1$ mode for $T_{p}$ $=1.9 \mathrm{eV}$ and $T_{\ell}=0.3 \mathrm{eV}$. (b) The same with noise added to the antenna (" $T_{\ell}$ " $=2.5 \mathrm{eV}$ ). The long-dashed line is the full Eq. (5) fitted to the data, with the solid line being the plasma component and the short-dashed line being the load noise filtered by the plasma.

same as the case for Fig. 4(a), except for the load being "hot." We added a white current source at the amplifier to increase the temperature of the load, effectively making $T_{\ell}$ $\approx 2.5 \mathrm{eV}$.

In Fig. 4(b), noise has been deliberately added to the antenna, corresponding to an effectively higher load temperature. The received spectrum has the same Lorentzian "plasma" component, but the dip and peak from the plasma "shorting" the load noise is more pronounced. This phasesensitive reflection and absorption of the load noise by the plasma effectively determines the antenna coupling coefficient $\mathcal{G}$. A full five-parameter fit of the received spectrum of Fig. 4(b) to Eq. (5) then gives $T_{p}=1.84 \mathrm{eV}, f_{m}=4.067 \mathrm{MHz}$, $\gamma_{m} / \omega_{m}=1.6 \times 10^{-3}, \mathcal{G}=0.43 \mathrm{pF}(0.39 \mathrm{~cm}$ in CGS $)$, and $T_{\ell}$ $=2.5 \mathrm{eV}$. For comparison, the standard dump diagnostic gives $T_{p}^{\text {dump }}=1.9 \mathrm{eV}$ for both Figs. 4(a) and 4(b).

Figure 4(b) demonstrates that the plasma temperature can be obtained in one measurement if the load is "noisy enough." Of course, if the emission from the load were to dominate the spectrum, the plasma component proportional to $T_{p}$ might be obscured. For Eq. (5) to be valid, the load noise must be uncorrelated with the plasma mode. Even a sine wave of constant amplitude that tracks the frequency of the analyzer (from a tracking generator) satisfies the criterion. Thus, the "noise" source in Fig. 4 can be a sine wave generator. This makes it more apparent that a reflection/ absorption measurement is being combined with an emission measurement. In practice, this is often the easiest and most effective source when using a swept analog analyzer.

We note that the damping rate $\gamma_{m}$ would be measurably smaller if the load "noise" drove the mode to large ampli- 


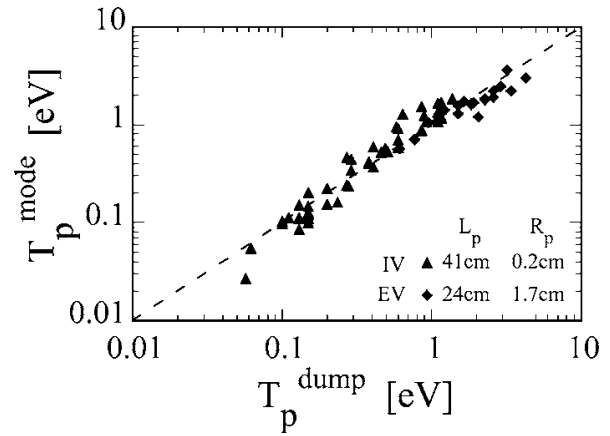

FIG. 5. Plasma temperature measured by mode analysis, compared to the standard dump temperature measurement. The triangles are from the IV apparatus and the diamonds are from the EV apparatus.

tude, because the wave would trap particles at the phase velocity and reduce the Landau damping. The thermal components [a solid curve in Fig. 4(b)] would then be viewed as small test waves in the presence of a larger-amplitude wave excited by the load noise.

Figure 5 displays the plasma temperature $T_{p}^{\text {mode }}$ obtained from the emission spectra near a mode versus the plasma temperature $T_{p}^{\mathrm{dump}}$ measured by dumping the plasma.

Data was taken for plasmas with a range of "geometric" parameters $\left(n, R_{p}, L_{p}\right)$ on both $\mathrm{EV}$ (circles) and IV (triangles), with varied amounts of plasma heating. Most of the values of $T_{p}^{\text {mode }}$ were obtained from a four-parameter fit to the emission spectra, together with a separate measurement of $\mathcal{G}$ for each $\left(n, R_{p}, L\right)$.

Figure 5 demonstrates that $T_{p}^{\text {mode }}$ and $T_{p}^{\text {dump }}$ agree well over a wide range of plasma parameters. We conclude that the mode emission spectra determines the temperature of plasma with about $\pm 15 \%$ error; here we note that the measurement of $T_{p}^{\text {dump }}$ itself typically has $\pm 15 \%$ error.

The mode analysis can be applied to any mode number $m_{z}$, but the error becomes larger as $m_{z}$ increases. This is because the coupling to the antenna becomes smaller as $m_{z}$ increases, and the "dip and peak" becomes smaller. Nevertheless, for the plasma of Fig. 4, we have measured the same $T_{p}^{\text {mode }}$ for $m_{z}=1,2,3$ within the $15 \%$ error.

\section{KINETIC THEORY}

One can alternately calculate $Z_{p}^{-1}$ using kinetic theory and compare to the measured spectrum over a broad frequency range encompassing several modes. A dressed test particle picture gives the analytical formula, assuming (1) the periodic boundary condition (infinite length), (2) that a plasma has constant density out to $R_{p}$, and (3) that kinetic Landau damping is the only significant damping mechanism (e.g., electron-neutral collisions are negligible).

The current flowing onto an antenna cylinder in response to a plasma charge is calculated by integrating the displacement current over the surface area $A=2 \pi R_{w} L_{w}$ of the antenna, as

$$
I=-i \omega \varepsilon_{0} \int_{A} E_{r} d S
$$

where $E_{r}$ is the radial electric field at the wall. The electrostatic potential $\phi$ is assumed to have spatial Fourier decomposition,

$$
\phi(r, z)=\sum_{m} \phi_{m}(r) \cos \left(k_{z} z\right), \quad 0<z<L p,
$$

with $k_{z} \equiv m \pi / L_{p}$, and $m \equiv m_{z}$; then

$$
E_{r}=-\sum_{m} \phi_{m}^{\prime}(r) \cos \left(k_{z} z\right) .
$$

Equation (6) can be written as

$$
I=-i \omega \varepsilon_{0} A \sum_{m} \phi_{m}^{\prime}\left(R_{w}\right) M_{m},
$$

where $\phi_{m}^{\prime}$ is a derivative of $\phi_{m}$ with respect to $r$ and $M_{m}$ represents the overlap of the mode $m$ with the antenna cylinder, as

$$
M_{m} \equiv \frac{1}{A} \int_{A} \cos \left(k_{z} z\right) d S \equiv \frac{L_{p}}{L_{w}} F_{m} .
$$

When $k_{z}\left(z_{2}-z_{1}\right) \equiv k_{z} L_{w} \ll 1$ and the antenna is located near the end of plasma, $M_{m}$ goes to unity. Thus, $M$ is a dimensionless "structure factor" that depends only on the location and size of the antenna cylinder in relation to the mode.

Conversely, when a potential $V$ is applied to the antenna, this excites various Fourier components, $\phi_{m}$, of magnitude

$$
\phi_{m}\left(R_{w}\right)=2 V M_{m} \text {. }
$$

In terms of the dimensionless plasma susceptibility, $\chi_{m}(\omega)=-R_{w} \phi_{m}^{\prime}\left(R_{w}\right) / \phi\left(R_{w}\right)$, we can write

$$
I=-i \omega \varepsilon_{0} A / R_{w} \sum_{m} \chi_{m}(\omega) M_{m}^{2} 2 V,
$$

and evaluate the plasma/antenna impedance, $Z_{p} \equiv V / I$, as

$$
\begin{aligned}
Z_{p}^{-1}(\omega) & =-i \omega 2 \frac{\varepsilon_{0} A}{R_{w}} \frac{L_{w}}{L_{p}} \sum_{m} \chi_{m}(\omega) M_{m}^{2} \\
& =-i \omega 4 \pi \varepsilon_{0} \frac{L_{w}^{2}}{L_{p}} \sum_{m} \chi_{m}(\omega) M_{m}^{2} .
\end{aligned}
$$

For a strong magnetic field, the susceptibility can be expressed as

$$
\chi_{m}(\omega)=G_{3} \frac{F(\omega)+G_{1}}{F(\omega)+G_{2}},
$$

with

$$
\begin{aligned}
& F(\omega) \equiv H(\omega) R_{p}\left(\frac{J_{0}^{\prime}\left[H(\omega) R_{p}\right]}{J_{0}\left[H(\omega) R_{p}\right]}\right), \\
& G_{1} \equiv k_{z} R_{p} \frac{I_{0}^{\prime}\left(k_{z} R_{w}\right) K_{0}^{\prime}\left(k_{z} R_{p}\right)-K_{0}^{\prime}\left(k_{z} R_{w}\right) I_{0}^{\prime}\left(k_{z} R_{p}\right)}{K_{0}^{\prime}\left(k_{z} R_{w}\right) I_{0}\left(k_{z} R_{p}\right)-I_{0}^{\prime}\left(k_{z} R_{w}\right) K_{0}\left(k_{z} R_{p}\right)}, \\
& G_{2} \equiv k_{z} R_{p} \frac{I_{0}\left(k_{z} R_{w}\right) K_{0}^{\prime}\left(k_{z} R_{p}\right)-K_{0}\left(k_{z} R_{w}\right) I_{0}^{\prime}\left(k_{z} R_{p}\right)}{K_{0}\left(k_{z} R_{w}\right) I_{0}\left(k_{z} R_{p}\right)-I_{0}\left(k_{z} R_{w}\right) K_{0}\left(k_{z} R_{p}\right)},
\end{aligned}
$$




$$
\begin{aligned}
& G_{3} \equiv k_{z} R_{w} \frac{K_{0}^{\prime}\left(k_{z} R_{w}\right) I_{0}\left(k_{z} R_{p}\right)-I_{0}^{\prime}\left(k_{z} R_{w}\right) K_{0}\left(k_{z} R_{p}\right)}{K_{0}\left(k_{z} R_{w}\right) I_{0}\left(k_{z} R_{p}\right)-I_{0}\left(k_{z} R_{w}\right) K_{0}\left(k_{z} R_{p}\right)}, \\
& H(\omega) \equiv k_{z} \sqrt{-\varepsilon_{3}}, \quad \varepsilon_{3}=1-\frac{1}{k_{z}^{2} \lambda_{\mathrm{D}}^{2}} X^{\prime}\left(y_{n}\right), \\
& y_{n}=\frac{v_{\phi}}{v_{\text {th }}}=\frac{1}{k_{z} \lambda_{\mathrm{D}}} \frac{\omega}{\omega_{p}} ;
\end{aligned}
$$

here $X\left(y_{n}\right)$ is the plasma dispersion function, normally denoted $Z\left(y_{n}\right)$. This analysis reduces to the simple pole of Eq. (4) for frequencies near a weakly damped mode.

The plasma/antenna coupling coefficient $\mathcal{G}$ can also be obtained from this analysis. In the limit of $T \rightarrow 0$, assuming that $\lambda_{\mathrm{D}} \ll r_{p}$ and that $k_{z} r_{w} \ll 1$, we find that

$$
\mathcal{G}=\frac{\left(4 \pi \varepsilon_{0}\right) L_{p} F_{m}^{2}}{1+x^{2} \ln ^{2}\left(R_{w} / R_{p}\right)}
$$

Here $x$ is a dimensionless quantity that satisfies the equation $x J_{1}(x) \ln \left(R_{w} / R_{p}\right)=J_{0}(x)$, and is related to the frequency of the plasma mode by $x=k_{z} R_{p}\left(\omega_{p}^{2} / \omega_{m}^{2}-1\right)^{1 / 2}$. For $R_{w} / R_{p} \gg 1$, one sees $x \approx \sqrt{2 / \ln \left(R_{w} / R_{p}\right)}$, which implies $\mathcal{G} \simeq\left(4 \pi \varepsilon_{0}\right) L_{p} F_{m}^{2} /[1$ $\left.+2 \ln \left(R_{w} / R_{p}\right)\right]$. Thus, $\mathcal{G}$ is the capacitance between the plasma and the wall, modified by the $\sin ^{2}(z)$ eigenfunction factor. All equations except Eq. (11) are valid in CGS or SI; in CGS, Eq. (11) would have no $4 \pi \varepsilon_{0}$.

Equation (11) presumes that the plasma column is penetrating the cylindrical antenna completely. When the antenna is at the end of the plasma and the plasma only partially penetrates the electrode, the plasma end point $z_{p}$ would replace the electrode end $z_{2}$ in estimating $F_{m}$.

\section{MULTIPLE MODE SPECTRA}

The $k_{z}=1,2,3, \ldots, m_{\theta}=0$ modes predicted by the kinetic theory are readily observed in practice, allowing the determination of $T_{p}$ as well as $n, R_{p}$, and $L_{p}$. Measuring the total antenna/cable/receiver complex impedance versus frequency is the main practical limitation; and the inadequacy of $z$-periodic kinetic theory for Landau damping of modes in short plasma columns is the main theoretical limitation.

\section{A. Cable resonances}

As we look at frequencies higher than $5 \mathrm{MHz}$, we need to calibrate for the coaxial cable resonance, since our amplifier load impedance $(690 \Omega)$ does not match the characteristic impedance of the handmade UHV coaxial cable $(90 \Omega)$, the voltage is resonantly amplified at near $20 \mathrm{MHz}$, where the wavelength of the signal in the cable becomes comparable to the cable length.

For some spectrum measurements, all of the coax cables other than the one connected to the antenna were terminated outside the vacuum chamber with $50 \Omega$ in order to avoid the transmission cable resonances that cause unwanted extra noise on an antenna above $10 \mathrm{MHz}$. The resistive damping of the mode caused by this $50 \Omega$ termination is $\gamma / \omega_{m}$ $\sim \mathcal{G} \omega_{m} R_{50 \Omega}$ [see Eq. (5)], estimated to be maximum about $10^{-3}$, which is comparable to the internal plasma damping for weakly damped plasma modes. This means that for lowtemperature plasmas where Landau damping is weak, even $50 \Omega$ terminations can double the plasma damping.

Figure 1(c) is the more complete circuit analog of Fig. 1(a). We now separate the load impedance $Z_{\ell}$ in Fig. 1(b) into three parts: (1) $Z_{n}$ is the impedance to the "next" cylinder, i.e., from the antenna through the neighbor cylinder, then terminated to ground with $50 \Omega$. (2) The transmission cable from the antenna to the receiver amplifier is modeled with a repeating LCR circuit. (3) The impedance $Z_{\ell}$ of the receiver amplifier is a resistor $R_{\ell}=690 \Omega$ and capacitor $C_{\ell}=60 \mathrm{pF}$ in parallel. This $C_{\ell}$ now only includes a $40 \mathrm{pF}$ inherent amplifier capacitance and the cable to the machine $(20 \mathrm{pF})$. The sum of the simple capacitances of $Z_{n}(2 \times 27 \mathrm{pF} \sim 50 \mathrm{pF})$, transmission cable $(80 \mathrm{pF})$, and receiver amplifier $Z_{\ell}(60 \mathrm{pF})$ adds up to the load capacitance $C_{\ell} \sim 190 \mathrm{pF}$ used in the simple model of $Z_{\ell}$ in Fig. 1. The existence of the impedance-mismatched coax cable brings two complications at higher frequencies in the voltage analysis; (1) that $V_{w}$ is no longer equal to $V_{a}$, and (2) that total impedance $Z_{\text {tot }}$ is different whether it is looked at from plasma current source $I_{p}\left(Z_{\mathrm{tot}}^{p}\right)$ or the load current source $I_{\ell}\left(Z_{\text {tot }}^{\ell}\right)$.

The voltages on either end of the coax cables are not necessarily the same. In order to understand the coax cable, we modeled the coax cable with repeating $\mathcal{L}, \mathcal{C}, \mathcal{R}$ components. Here $\mathcal{L}, \mathcal{C}, \mathcal{R}$ refers to the inductance, capacitance, and resistance per unit length of the cable. We define $V_{w p}$ as the voltage induced by the plasma on the wall side and $V_{a p}$ as a voltage induced by the plasma, transmitted through the cable, and measured at amplifier side, as shown in Fig. 1(c). This $V_{w p}$ and $V_{a p}$ equals $V_{w}$ and $V_{a}$, respectively, when there is no load-generated noise $\left(I_{\ell}=0\right)$.

We calculate the coax cable voltage ratio $\xi$, which is defined as the ratio of the two voltages $V_{a p}$ and $V_{w p}$. Here $\xi$ depends on the cable impedance $Z_{0}(\approx 90 \Omega)$, the cable length $L_{T}$ and propagation constant $\Gamma$, and the receiver impedance $Z_{\ell}$, as ${ }^{24}$

$$
\xi(\omega) \equiv \frac{V_{a p}(\omega)}{V_{w p}(\omega)}=\left|\frac{Z_{\ell}}{Z_{\ell} \cosh \left(\Gamma L_{T}\right)+Z_{0} \sinh \left(\Gamma L_{T}\right)}\right|,
$$

where

$$
\begin{aligned}
& \Gamma \equiv \sqrt{\omega^{2} \mathcal{L C}+i \omega \mathcal{R C}}, \\
& Z_{0} \equiv \sqrt{\frac{\mathcal{R}+i \omega \mathcal{L}}{i \omega \mathcal{C}}} .
\end{aligned}
$$

Figure 6 shows the calculated $\xi(f)$ from Eq. (12), for our apparatus. Here $Z_{\ell}$ consists of a $690 \Omega$ resistor in parallel with a $60 \mathrm{pF}$ capacitor $\left(Z_{\ell}=690 \Omega / / 60 \mathrm{pF}\right)$.

The coax cable that we used is a handmade coax cable with $0.33 \mathrm{~mm}$ central copper wire and $0.46 \mathrm{~cm}$ inner diameter copper tube as a shield, insulated with an alumina tube in between. This coax is well modeled with the $\mathcal{L}, \mathcal{C}, \mathcal{R}$ parameters in Fig. 6. These parameters were obtained by comparing the resulting calculated impedance to reflection/ absorption measurements of the cable. The central copper cable itself has a dc resistance of only $0.3 \Omega / \mathrm{m}$, so $\mathcal{R}$ presumably represents $\mathrm{rf}$ dissipation in the alumina dielectric. 


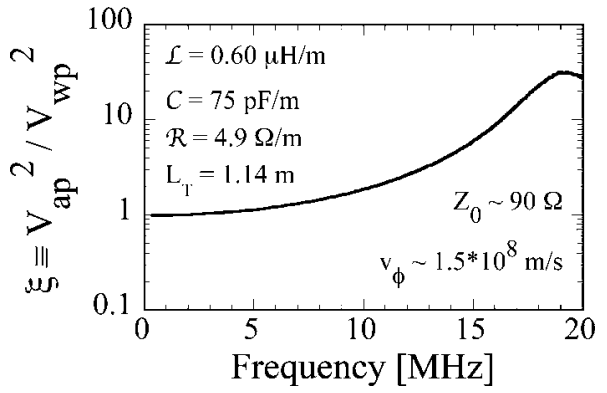

FIG. 6. Calculated voltage $V_{a p}$ induced by plasma on the amplifier, normalized by the voltage $V_{w p}$ induced by the plasma directly on the antenna wall versus frequency.

The impedance of our receiver $Z_{\ell} \sim 690 \Omega / / 60 \mathrm{pF}$ was separately obtained by a reflection/absorption measurement.

Here $V_{a p}$ is resonantly amplified by the transmission cable, causing $V_{w p}$ to have a peak at $19 \mathrm{MHz}$, with a peak gain of $36 \times$ in power. This transmission cable resonance is advantageous for our measurement, because the electrostatic fluctuations of plasmas have smaller spectral densities at higher frequencies. Using a lower $Z_{\ell}$ that matches $Z_{0}$ avoids this cable resonance, but it would give less of a signal.

\section{B. Comparison to theory}

Applying the Nyquist theorem to the circuit shown in Fig. 1(c), we get

$$
\begin{aligned}
\frac{V_{a}^{2}}{d f}(\omega) & =\xi^{2} \frac{I_{p}^{2}}{d f}\left|Z_{\mathrm{tot}}^{p}\right|^{2}+\frac{I_{\ell}^{2}}{d f}\left|Z_{\mathrm{tot}}^{\ell}\right|^{2} \\
& =4 T_{p} \operatorname{Re}\left\{Z_{p}^{-1}\right\} \frac{V_{a p}^{2}}{V_{w p}^{2}}\left|Z_{\mathrm{tot}}^{p}\right|^{2}+4 T_{\ell} \operatorname{Re}\left\{Z_{\ell}^{-1}\right\}\left|Z_{\mathrm{tot}}^{\ell}\right|^{2},
\end{aligned}
$$

where

$$
\begin{aligned}
& \left(Z_{\text {tot }}^{p}\right)^{-1}=Z_{p}^{-1}+Z_{n}^{-1}+Z_{\text {in }}^{-1}, \\
& Z_{\text {in }}=Z_{0}\left(\frac{Z_{\ell} \cosh \left(\Gamma L_{T}\right)+Z_{0} \sinh \left(\Gamma L_{T}\right)}{Z_{0} \cosh \left(\Gamma L_{T}\right)+Z_{\ell} \sinh \left(\Gamma L_{T}\right)}\right),
\end{aligned}
$$

and $Z_{\text {tot }}^{p}$ is a total parallel impedance of $Z_{p}, Z_{n}$, and $Z_{\text {in }}$ that plasma current $I_{p}$ sees [Fig. 1(c)]. Here $Z_{n}$ is the impedance to the next cylinder and $Z_{\text {in }}$ is an input impedance of the transmission cable terminated with $Z_{\ell}$ at the end. ${ }^{24}$ The image charge induces a voltage $V_{w p}=I_{p}\left|Z_{\text {tot }}^{p}\right|$ at the antenna, then calibrated for the transmission cable by $\xi$.

The voltage measured at amplifier $V_{a}^{2}$ has two components. One is the fluctuating voltage induced by plasma current $I_{p}$ running through the total impedance seen from plasma $Z_{\text {tot }}^{p}[$ see Eq (14)], corrected for the cable resonance voltage ratio $\xi^{2}$. The other is the load noise current running through total impedance $Z_{\text {tot }}^{\ell}$ that the load current $I_{\ell}$ sees, where

$$
\left(Z_{\text {tot }}^{\ell}\right)^{-1}=Z_{\ell}^{-1}+Z_{\text {out }}^{-1}
$$

and $Z_{\text {out }}$ is an output impedance of the transmission cable terminated with $1 / 1 / Z_{p}+1 / Z_{n}$ at the antenna side and is calculated as

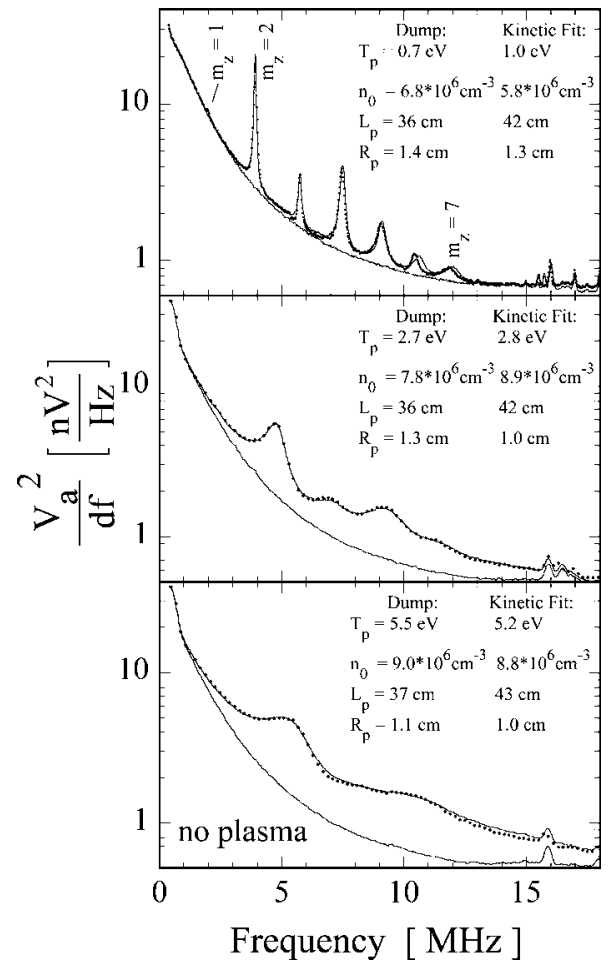

FIG. 7. Measured emission spectral density on the receiver amplifier (dots) for $T_{p}=0.7,2.7$, and $5.4 \mathrm{eV}$. The lower curves are receiver noise spectra without plasma. Upper curves show the kinetic theory of Eq. (13),.., (10), with $T_{p}, n_{0}, L_{p}, R_{p}$ as fit parameters.

$$
Z_{\text {out }}=Z_{0}\left(\frac{\frac{1}{1 / Z_{p}+1 / Z_{n}} \cosh \left(\Gamma L_{T}\right)+Z_{0} \sinh \left(\Gamma L_{T}\right)}{Z_{0} \cosh \left(\Gamma L_{T}\right)+\frac{1}{1 / Z_{p}+1 / Z_{n}} \sinh \left(\Gamma L_{T}\right)}\right) .
$$

Figure 7 shows the voltage power spectra on the receiver amplifier. The measured broad spectra show stunningly close agreement with the predictions of kinetic theory.

For $T_{p}^{\text {dump }}=0.7 \mathrm{eV}$, we can see modes up to $m_{z}=7$. The $m_{z}=1$ mode has small spectral density because the antenna is centered on a node. As the temperature increases to $2.7 \mathrm{eV}$, each mode frequency increases somewhat due to thermal pressure, the damping increases strongly due to Landau damping, and the broad interpeak spectrum increases. At $T_{p}^{\text {dump }}=5.4 \mathrm{eV}$, the modes are damped within one cycle, and the broad spectrum becomes dominant.

Lower lines on each spectrum are the measured fluctuation spectrum without plasma, representing receiver noise.

For $T_{p}^{\text {dump }}=2.7$ and $5.4 \mathrm{eV}$, we used a spectrum analyzer with $\mathrm{BW}=300 \mathrm{kHz}$, video filter $=100 \mathrm{~Hz}$, scan time $=0.5 \mathrm{~s}$, and scan range $=20 \mathrm{MHz}$. For $T_{p}^{\text {dump }}=0.7 \mathrm{eV}$, we used $\mathrm{BW}$ $=100 \mathrm{kHz}$ and took four separate scans over $5 \mathrm{MHz}$ to get a 0 to $18 \mathrm{MHz}$ spectrum. Spectral density at each frequency was averaged over 16 shots. For $T_{p}=2.7$ and $5.5 \mathrm{eV}, 10 \%$ of the measured data are shown in dots to make the fit curves visible. 
Now we explain how the temperature was determined through fit to kinetic theory. We rewrite Eq. (13) to make $Z_{p}^{-1}$ more explicitly visible:

$$
\begin{aligned}
& \frac{V_{a}}{d f}(\omega)=4 T_{p} \operatorname{Re}\left\{\mathbf{Z}_{\mathbf{p}}^{-1}\right\} \xi^{2}\left|\mathbf{Z}_{\mathbf{p}}^{-1}+Z_{n}^{1}+Z_{\text {in }}^{-1}\right|^{-2}+\frac{I_{\ell}^{2}}{d f} \operatorname{Re}\left\{Z_{\ell}^{-1}\right\} \\
& \begin{array}{l}
\times \mid Z_{\ell}^{-1}+Z_{0}^{-1} \\
\times\left(\frac{\frac{1}{\mathbf{Z}_{\mathbf{p}}^{-1}+Z_{n}^{-1}} \cosh \left(\Gamma L_{T}\right)+Z_{0} \sinh \left(\Gamma L_{T}\right)}{Z_{0} \cosh \left(\Gamma L_{T}\right)+\frac{1}{\mathbf{Z}_{\mathbf{p}}^{-1}+Z_{n}^{1}} \sinh \left(\Gamma L_{T}\right)}\right)^{-1} \mid-.
\end{array}
\end{aligned}
$$

We directly measure all quantities on the right-hand side such as $Z_{\ell}, \xi, Z_{n}^{-1}, Z_{i n}^{-1}, \Gamma, L_{T}, Z_{0}, I_{\ell}^{2} / d f . Z_{p}$ is calculated and shown with bold in the equation. The measured spectrum without plasma was used to calculate the receiver amplifier noise $I_{r}^{2} / d f$ in Eq. (13). Here $Z_{p}$ is calculated by the kinetic theory and has four parameters, $T_{p}, n_{0}, L_{p}, R_{p}$.

Thus, the right-hand side of Eq. (16) has four parameters, and fitting to the measured spectral data determines all four parameters.

When the modes were very weakly damped, as for $T_{p}$ $=0.7 \mathrm{eV}$, the fitting routine did not readily converge to the correct answer, and we needed to start with the parameters that were close to the "right" ones. In contrast, the fit converged easily for the spectrum with strong damping of the modes.

At all three temperatures in Fig. 7, the kinetic theory calculates the emission spectrum so well that we are able to determine the plasma parameters, $T_{p}, n_{0}, L_{p}$, and $R_{p}$, from the broad emission spectrum. This means that we can determine all four plasma parameters with an emission data digitized over $1 / 2 \pi \gamma_{\min } \mathrm{s}$, which allows us to have enough frequency resolution. Here, $\gamma_{\min }$ is the damping of the mode that has the minimum damping of all $m_{z}$.

Here, $L_{p}^{\mathrm{kin}}$ obtained from the fit is consistently $17 \%$ higher than $L_{p}^{\text {dump }}$, obtained from the measured profile, particle number, and solving iteratively the Poisson and Boltzmann equations for the self-consistent density and potential. This is due to the "end effect" on Landau damping in the finite length system, and will be discussed later.

\section{Number fluctuation spectra}

We can also calculate the charge number fluctuation spectrum $\delta N_{w}^{2} / d f$ from the measured $V_{a}^{2} / d f$ on the antenna. All the experimental complications like the receiver noise spectrum $V_{a}^{2}(f) / d f$ are removed from $\delta N_{w}^{2} / d f$, so it is easier to compare with theory directly.

We calculate the $\delta N_{w}^{2} / d f$ from $V_{a}^{2} / d f$ as follows:

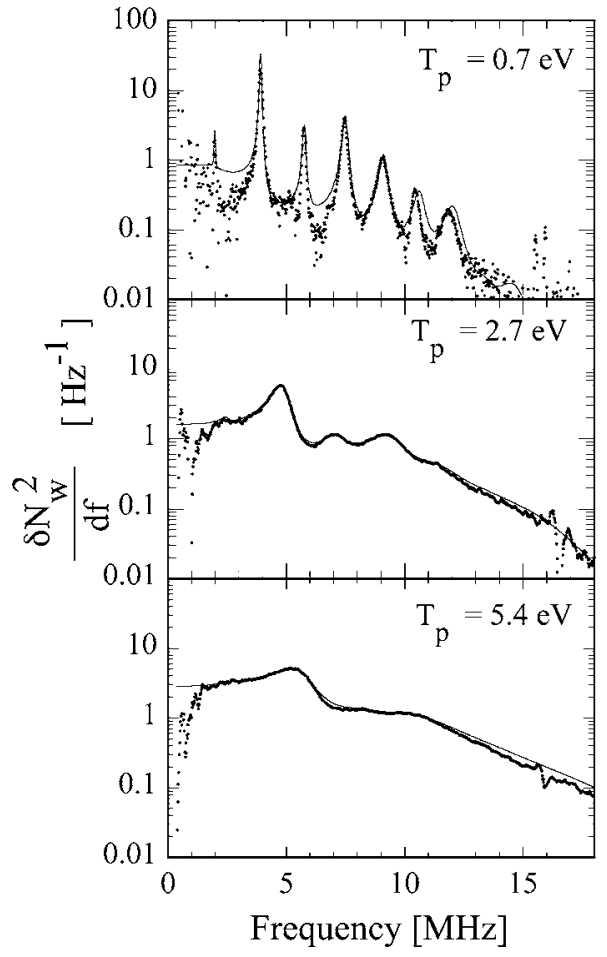

FIG. 8. Charge number fluctuation spectra for the data of Fig. 7, with kinetic theory fits.

$$
\frac{\delta N_{w}^{2}}{d f}=\frac{I_{p}^{2}}{d f} \frac{1}{\omega^{2} e^{2}}=\frac{\Delta V_{a}^{2}}{d f} \xi^{-2}\left|Z_{\mathrm{tot}}^{p}\right|^{-2} \frac{1}{\omega^{2} e^{2}},
$$

where $\Delta V_{a} / d f$ is a difference of the voltage spectrum $V_{a}^{2} / d f$ with and without plasma.

Here $\Delta V_{a} / d f$ is corrected for the coaxial cable resonance $\xi$, converted to the current by $\left|Z_{\text {tot }}^{p}\right|^{-2}$ then finally divided by $\omega^{2} e^{2}$ to obtain the charge fluctuation $\delta N_{w}^{2} / d f$. Note that $\left|Z_{\text {tot }}^{p}\right|^{-2}$ includes $Z_{p}^{-1}$, and we used the $Z_{p}^{-1}$ that was obtained by the previous fit.

Figure 8 displays the spectrum of $\delta N_{w}^{2} / d f$ for the thermal emission measurements of Fig. 7. At $0.7 \mathrm{eV}$, we see that measurement and theory agree to within $30 \%$ over a wide range in frequency, encompassing seven modes; and that the dynamic range for the amplitude is about three orders of magnitude. Moreover, correspondence is obtained at all temperatures, here ranging over a factor of 20. Our detection electronics loses gain below $1.5 \mathrm{MHz}$ because the amplifier becomes resistive below $f_{R C}=1 / 2 \pi R C \sim 1.2 \mathrm{MHz}$, with gain proportional to frequency squared.

Figure 9 displays the plasma temperature $T_{p}^{\text {kin }}$ obtained from the emission spectra fitted to the kinetic theory calculation, versus the plasma temperature $T_{p}^{\text {dump }}$ measured by dumping the plasma. Transmission cables were terminated with $50 \Omega$ for solid diamonds $(\diamond)$ and were shorted for all the open symbols.

The fit gives accurate temperatures for $36 \mathrm{~cm}$ plasmas, even when the cables were not terminated with $50 \Omega$. This is because the spectrum below $10 \mathrm{MHz}$ dominates, and the fit is not significantly affected by the transmission cable resonance above $10 \mathrm{MHz}$. For shorter plasmas, $T_{p}^{\mathrm{kin}}$ is systematically lower than $T_{p}^{\text {dump }}$. The kinetic theory assumes a periodic 


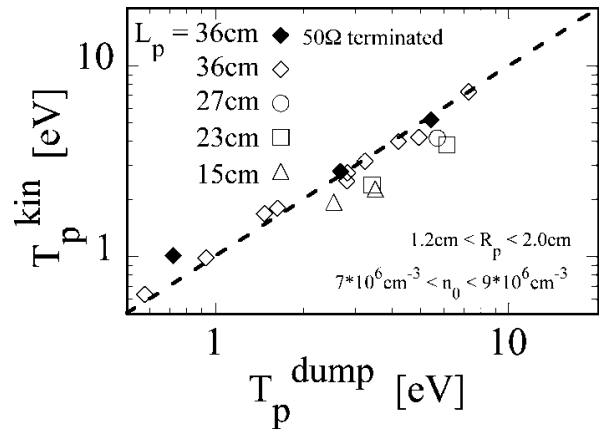

FIG. 9. Temperature obtained with kinetic theory versus temperature obtained with dump. The length varies from 15 to $36 \mathrm{~cm}$.

$z$-boundary condition, and therefore significantly overestimates the Landau damping for finite length plasmas.

Fitting the kinetic theory to broad emission spectra determines the plasma temperature $T_{p}$, as well as $n_{0}, R_{p}$, and $L_{p}$. We find that $T_{p}$ is accurately determined for long plasmas (with $L_{p} / R_{p} \geqslant 20$ ); but the fit is less satisfactory for shorter plasmas, because kinetic theory with periodic $z$ boundaries does not properly model Landau damping in finite-length plasmas.

Figure 10 is an example of such a case, where the kinetic theory at the actual $T_{p}$ predicts much broader peaks (stronger damping) than the measured peak width. The solid line is a kinetic theory calculation at the actual plasma temperature, showing that $\gamma$ is $2 \times$ too large. The dashed line is a best "fit," giving temperatures about $2 \times$ too small. This fit also gives $n_{0} R_{p}^{2}$ about $1.3 \times$ too large, and $N_{\text {tot }}$ about $1.5 \times$ too large.

That is, kinetic theory overestimates the Landau damping by a factor of 2 for this length. The fitting routine matches the damping by lowering the temperature.

Landau damping is predicted to depend on the mode phase velocity $v_{\phi} \equiv \omega / k_{z}$; but the definition of $k_{z}$ for finite length plasmas is problematical. We define an "effective wave number" $k_{z}^{\text {eff }}$ as that which gives the measured mode frequency $\omega_{m}$ in linear (infinite-length) wave theory. This $k_{z}^{\text {eff }}$

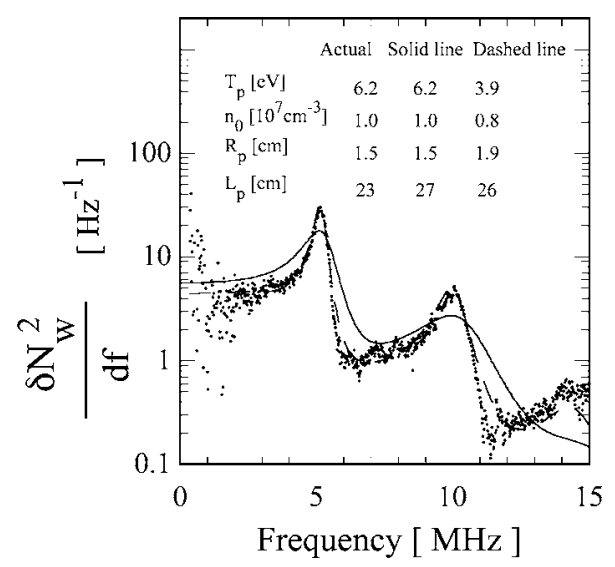

FIG. 10. Effects of overestimated Landau damping in kinetic theory for damping and Landau damping for the shorter $\left(L_{p}=23 \mathrm{~cm}\right)$ plasma. The solid line is a kinetic theory calculation at the actual $T_{p}=6.2 \mathrm{eV}$, using $L_{p}^{k}$ $=27 \mathrm{~cm}$ to match the mode frequencies. The broken line is a kinetic theory fit, giving $T_{p}^{\mathrm{kin}}=3.9 \mathrm{eV}$ with compensating errors in $n_{0}, L_{p}, R_{p}$.

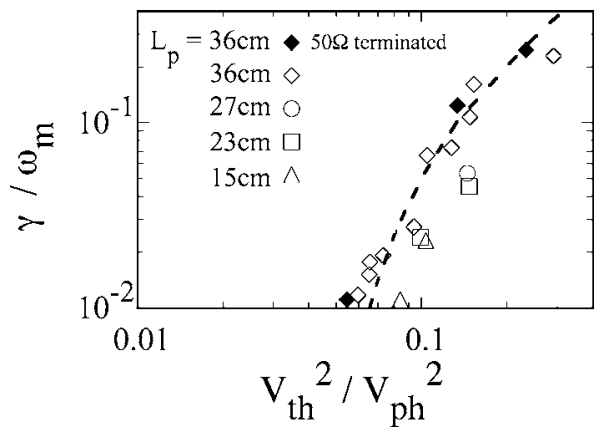

FIG. 11. Damping $\gamma$ normalized by the mode frequency $\omega_{m} \mathrm{vs}\left(v_{\mathrm{th}} / v_{\phi}\right)^{2}$.

is then used to calculate $v_{\phi}$, as $v_{\phi}=\omega_{m} / k_{z}^{\text {eff }}$. This $k_{z}^{\text {eff }}$ is typically $20 \%$ lower than $m \pi / L_{p}$, as was first discussed by Spencer. ${ }^{25}$

Figure 11 shows the predicted and observed damping $\gamma$ (normalized by mode frequency $\omega_{m}$ ) versus thermal velocity $v_{\text {th }}^{2}$ over phase velocity $v_{\phi}^{2} \equiv\left(\omega / k_{z}^{\text {eff }}\right)^{2}$ squared. The symbols show experimental data for various $L_{p}$. Here, $\gamma$ is the halfwidth at half-maximum of the $\delta N^{2}$ spectrum, measured on the higher side of the mode. We picked the mode that has the largest amplitude out of the spectra that were used for Fig. 9 in order to determine $\gamma$ and $\omega_{m}$. For long plasmas, the agreement between theory and experiment is quite close; for short plasmas, the theory predicts larger damping than is observed.

In the theory, $v_{\mathrm{th}}^{2} / v_{\phi}^{2}$ tells where the wave phase velocity is located in the electron velocity distribution, and thus determines the Landau damping. For shorter plasmas, damping is systematically low or else $k_{z}^{\text {eff }}$ is low compared to the theory. Clearly, end effects play a substantial role for short plasmas. As an extreme example, a football-shaped plasma in a harmonic potential theoretically has no damping ${ }^{26}$ for modes corresponding to $m_{z}=1$ and 2. Here, no "adjustment" of $k_{z}$ in infinite-length theory is likely to eliminate the damping.

Overall, our four-parameter fit to theory determines $T_{p}, n, L_{p}, R_{p}$ to within $30 \%$ accuracy for the long plasmas, where kinetic theory calculates the damping correctly. For short plasmas, the fit temperature deviates by $50 \%$ because the damping differs from theory by up to $5 \times$.

\section{TOTAL FLUCTUATION ANALYSIS USING THERMODYNAMIC THEOREM}

All errors associated with the details of the spectral shape can be avoided by looking at the total frequencyintegrated fluctuation level $\delta N_{w}^{2}$ induced on the wall antenna. Thermodynamic theory ${ }^{27}$ calculates $\delta N_{w}^{2}$ for any given $T_{p}, n_{0}, R_{p}$, and geometry. This thermodynamic argument is more general than kinetic theory, in the sense that it does not invoke the shape of the spectrum.

The total fluctuation number is found to be 


$$
\begin{aligned}
\frac{\delta N^{2}}{N}= & \frac{4 \lambda_{\mathrm{D}}^{2} L_{w}}{R_{p}^{2} L_{p}} \\
& \times \sum_{n=1} M_{m}^{2} \frac{-\hat{k}_{z} I_{0}\left(k_{z} R_{p}\right) I_{1}\left(\hat{k}_{z} R_{p}\right)+k_{z} I_{0}\left(\hat{k}_{z} R_{p}\right) I_{1}\left(k_{z} R_{p}\right)}{I_{0}\left(k_{z} R_{w}\right) D},
\end{aligned}
$$

with

$$
\begin{aligned}
D \equiv & \hat{k}_{z} I_{1}\left(\hat{k}_{z} R_{p}\right)\left\{I_{0}\left(k_{z} R_{p}\right) K_{0}\left(k_{z} R_{w}\right)-I_{0}\left(k_{z} R_{w}\right) K_{0}\left(k_{z} R_{p}\right)\right\} \\
& -k_{z} I_{0}\left(\hat{k}_{z} R_{p}\right)\left\{I_{1}\left(k_{z} R_{p}\right) K_{0}\left(k_{z} R_{w}\right)+I_{0}\left(k_{z} R_{w}\right) K_{1}\left(k_{z} R_{p}\right)\right\}, \\
\hat{k}_{z}^{2} \equiv & k^{2}+\frac{1}{\lambda_{\mathrm{D}}^{2}} \quad \text { and } \quad \lambda_{\mathrm{D}}^{2} \equiv\left[4 \pi \varepsilon_{0}\right] \frac{T_{p}}{4 \pi n_{0} e^{2}} .
\end{aligned}
$$

Here, $\delta N^{2}$ is normalized by the total number of the electrons $N$ inside the antenna region.

This rather complicated function is well approximated by a simpler form,

$$
\frac{\delta N_{w}^{2}}{N_{w}} \cong \frac{\alpha_{1}}{1+\alpha_{2}\left(\frac{R_{p}}{\lambda_{\mathrm{D}}}\right)^{2}} .
$$

This form shows that the fluctuation level is a constant in the high-temperature limit $\left(R_{p} / \lambda_{\mathrm{D}} \ll 1\right)$, and is proportional to the temperature at low temperatures $\left(R_{p} / \lambda_{\mathrm{D}} \gg 1\right)$.

In the limit $T_{p} \rightarrow \infty$, we obtain

$$
\begin{aligned}
\frac{\delta N_{w}^{2}}{N_{w}} & \rightarrow \alpha_{1}=2 \frac{L_{w}}{L_{p}} \sum_{n=1} M_{m}^{2} \frac{I_{0}^{2}\left(k_{z} R_{p}\right)-I_{1}^{2}\left(k_{z} R_{p}\right)}{I_{0}^{2}\left(k_{z} R_{w}\right)} \\
& \rightarrow 1-\frac{L_{w}}{L_{p}} \quad \text { for large } \frac{L_{w}}{R_{w}} \\
& =0.47 \quad \text { for our geometry with } L_{p}=42.3 \mathrm{~cm}, \\
L_{w} & =7.76 \mathrm{~cm}, \quad R_{w}=3.81 \mathrm{~cm} .
\end{aligned}
$$

When $L_{p} \gg L_{w} \gg R_{w}$, fluctuation is equal to 1 , and this represents the uncorrelated density fluctuations of an ideal gas in a cylinder. This high-temperature limit was simulated and compared to experiments. ${ }^{4}$ In this limit, the image charge induced on the antenna wall is equal to the number of electrons in the antenna.

In the low-temperature limit, where $k \lambda_{\mathrm{D}} \ll 1$,

$$
\begin{aligned}
\frac{\delta N_{w}^{2}}{N_{w}} \rightarrow & \frac{\alpha_{1}}{\alpha_{2}}\left(\frac{\lambda_{\mathrm{D}}}{R_{p}}\right)^{2}=4 \frac{L_{w}}{L_{p}}\left(\frac{\lambda_{\mathrm{D}}}{R_{p}}\right)^{2} \sum_{n=1} M_{m}^{2} \frac{I_{0}\left(k_{z} R_{p}\right)}{I_{0}\left(k_{z} R_{w}\right)} \\
& \times \frac{1}{-I_{0}\left(k_{z} R_{p}\right) K_{0}\left(k_{z} R_{w}\right)+K_{0}\left(k_{z} R_{p}\right) I_{0}\left(k_{z} R_{w}\right)} \\
\rightarrow & \frac{1-L_{w} / L_{p}}{\frac{1}{2} \ln \left(\frac{R_{w}}{R_{p}}\right)}\left(\frac{\lambda_{\mathrm{D}}}{R_{p}}\right)^{2} \text { for } \frac{L_{w}}{R_{w}} \gg 1 .
\end{aligned}
$$

Therefore,

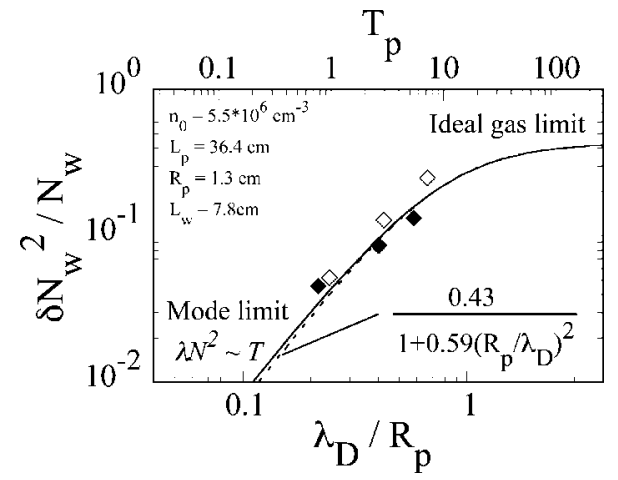

FIG. 12. The total fluctuation square normalized by the total number versus the Debye length normalized by the radius of the plasma. The solid line is the result of a thermodynamic argument. The broken line is the plot of the approximated form. Solid diamonds are measured data with transmission cables terminated with $50 \Omega$, and open diamonds are with shorted cables.

$$
\begin{aligned}
\alpha_{2} & =\frac{1}{2} \ln \left(\frac{R_{w}}{R_{p}}\right) \quad \text { for } \frac{L_{w}}{R_{w}} \gg 1 \\
& \approx 0.54 \text { for our geometry. }
\end{aligned}
$$

As the temperature decreases, Debye shielding supresses the random particle fluctuations. At low temperatures, collective effects become dominant, and the total fluctuation is proportional to $T_{p}$ in $\lambda_{\mathrm{D}}^{2}$.

Figure 12 shows the predicted and measured total fluctuation level versus temperature $T_{p}$ or $\lambda_{\mathrm{D}}$. Close agreement is obtained over a range of 10 in $T_{p}$. The solid line is the thermodynamic calculation of Eq. (18) using the (measured) parameters shown. The broken line is Eq. (19) with best fit to Eq. (18), giving $\alpha_{1}=0.43$ and $\alpha_{2}=0.59$. The figure shows that Eq. (19) describes Eq. (18) well with two fitting parameters. Here, $L_{w} / R_{w} \approx 2$ is not large enough to use the infinite length limit form of $\alpha_{1}$ and $\alpha_{2}$ described above.

Experimental points (diamonds) were obtained by first calculating $\delta N_{w}^{2} / d f$ from Eq. (17), using the same spectra in Figs. 7 and 8 and then integrated $\delta N_{w}^{2} / d f$ from 0 to $18 \mathrm{MHz}$, with two assumptions. First, we assumed $\left|Z_{p}\right| \gg\left|Z_{\ell}\right|$ so that $1 / Z_{p}$ becomes negligible, allowing us to calculate $\delta N_{w}$ without using the kinetic theory calculation of $Z_{p}$. This is a good assumption, except for the frequencies near a very weakly damped mode. For example, the minimum $Z_{p}(\sim 1200 \Omega)$ of Fig. 7 is given at the peak of the $m_{z}=2$ mode for $T$ $=0.7 \mathrm{eV}$, which is still larger than $Z_{\ell}$. Second, we also assumed that below $2 \mathrm{MHz}$, the fluctuation spectral density is flat with the value at $2 \mathrm{MHz}$. This is required since our receiver loses gain below $2 \mathrm{MHz}$, as the input impedance becomes resistive rather than capacitive. Here $N_{w}$ is obtained by $N_{w}=N_{\text {tot }} L_{w} / L_{p}$, with $N_{\text {tot }}$ and $L_{p}$ determined by a dump measurement. Integrating up to $18 \mathrm{MHz}$ is sufficient, since $\delta N_{w}^{2} / d f N_{w}<10^{-8}$ at $18 \mathrm{MHz}$.

The measured total fluctuations with properly terminated cables $(\checkmark)$ are within $20 \%$ agreement with a calculation of the thermodynamic argument. The measured fluctuations with shorted cables still show fair agreement with the thermodynamic calculation, despite the fact that the spectrum has an extra fluctuation above $10 \mathrm{MHz}$. For example, for the 


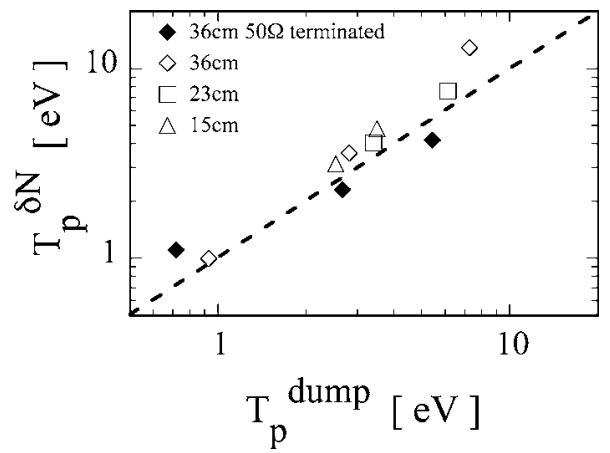

FIG. 13. Temperature obtained from the measured total fluctuation, compared with the thermodynamic argument versus temperature obtained with dump. The length varies from 15 to $36 \mathrm{~cm}$. All the transmission cables that are connected to cylinders except for the one connected to the antenna were terminated with $50 \Omega$ for solid diamonds. The same cylinders were shorted for open symbols.

open diamond at $\lambda / R_{p}=0.67$, shorted cables give a $20 \%$ increase in $\delta N^{2}$ above $10 \mathrm{MHz}$.

The measured total fluctuation compared to the theory line of Eq. (18) determines the temperature of the plasma. This total fluctuation serves as a "robust" temperature diagnostic because this method does not depend on the shape of the spectrum. However, $R_{p}, n_{0}$, and $L_{p}$ need to be predetermined in order to calculate the predicted total fluctuation, and all spectral biases, such as the cable resonance $\xi(\omega)$ must be known over the full frequency range.

Figure 13 shows the temperature $T_{p}^{\delta N}$ of the plasma obtained from the measured total fluctuation, versus $T_{p}^{\text {dump }}$. Solid symbols are data taken with the properly terminated cables. Open symbols are plasmas with shorted cables. Open symbols estimate $T_{p}^{\delta N}$ consistently higher because of the extra fluctuations. Nevertheless, this temperature diagnostics determines temperature to within 50\% accuracy and is independent of the form of the damping.

\section{DISCUSSION}

We have developed three different nonperturbative strategies to determine the plasma temperature.

The first method focuses on the spectrum near a mode, and approximates $Z_{p}^{-1}$ with a simple pole. This $Z_{p}$ does not presume any particular cause of the damping, other than that it is weak. This diagnostic determines $T_{p}, T_{\ell}, \omega_{m}, \gamma_{m}$, and $\mathcal{G}$ from the spectrum. This method has proven to be fairly robust in a "quiet" plasma environment. However, we note that each mode represents one of freedom out of $10^{9}$, and so it could easily be excited by an external signal (e.g., noise on a confining electrode) at the mode frequency.

The second method utilizes the emission spectrum over a broad frequency range, fitting it to kinetic theory. Kinetic theory implicitly assumes that Landau damping is the only damping mechanism, and neglects end corrections to this damping. This theory matches the measured spectra extremely well, if the temperature is large enough that Landau damping is dominant, and if the plasma is long compared to the radius. This diagnostic determines all four fitting parameters, giving $T_{p}, n_{0}, k_{z}, R_{p}$.

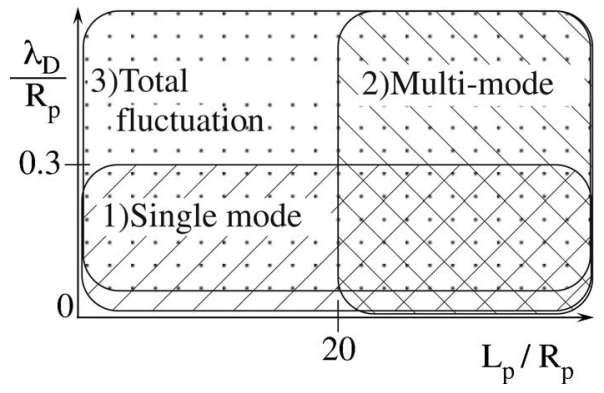

FIG. 14. Applicability of the three methods in different plasma regimes.

The third method utilizes the total frequency-integrated number fluctuation, and requires prior knowledge of $n_{0}, L_{p}, R_{p}$. The frequency integral requires a complete spectrum that is corrected for cable resonance. A comparison with a thermodynamic calculation determines the plasma temperature, so no assumptions are made as to the form of damping.

Figure 14 summarizes the plasma regimes for which each method works. As long as the chosen mode is weakly damped, the single mode analysis is robust, and a single emission spectrum determines the temperature. When the temperature is high enough that the mode spectrum deviates from a Lorentzian, one can use multimode scan and compare to the kinetic theory calculation if the plasma is long. For short plasmas at high temperatures, one can use thermodynamics to calculate the total fluctuation; but this requires a separate determination of $n_{0}, L_{p}, R_{p}$.

We will now compare the temperature diagnostics using thermal fluctuations to three diagnostics that have been developed in the past.

The "Magnetic Beach Analyzer" that determines $T_{\perp}$, and the "Evaporative Method" that determines $T_{\|}$, were developed in the 1970s. These two methods employ the direct measurement of electron velocities, and this requires the plasma to be "dumped." The "Magnetic Beach Analyzer" has the advantage that one can determine $T_{\perp}$ at different radial locations, whereas thermal fluctuations provide only a "global" temperature. The disadvantage of the magnetic beach analyzer is that it typically takes about 80 shots of plasmas to have a decent signal-to-noise ratio.

In the 1990s, a third method was developed, based on the thermal shifts of mode frequencies. The frequencies of the center-of-mass mode and quadrupole mode are compared with the frequencies calculated theoretically for a harmonic potential. ${ }^{26,28,29}$ This method has a big advantage compared to thermal fluctuations because one can excite the mode, and therefore it is useful in noisy environments. However, this method relies on the theory for a plasma in a harmonic potential, and therefore suffers when the trap potential is not harmonic. One needs to either use hyperbolic electrodes or to apply stepped confinement voltages on several cylinders in order to create a harmonic potential. It was experimentally shown that this method fails when the plasma is big (long or "fat"), where the harmonic potential is no longer a good approximation. ${ }^{29,30}$ This limitation is analogous to the limitation of the kinetic theory. In our calculation of thermal 
fluctuations using kinetic theory, we assumed that the plasma is infinitely long, and the calculation failed when the plasma is short such that end effects become important. This points out that the theory of plasma modes needs further development in the regimes in between infinite-length and shortspheroidal plasmas.

On the other hand, our thermal single mode analysis is a very general approach, in the sense that it only uses Nyquist's theorem. Thermal mode analysis does not require a calculation of the plasma dielectric, and the geometrical factors are self-calibrated experimentally because the coupling coefficient $\mathcal{G}$ is measured at the same time as we measure the mode spectrum. Though not demonstrated here, the thermal mode analysis will presumably also work in quadrupole traps, regardless of the size of the plasmas. Since it can utilize the signal from any interior electrode, the setup effort is minimal; and being entirely passive, the diagnostic does not interfere with other experimental processes.

\section{ACKNOWLEDGMENTS}

We thank Professor R. E. Pollock for his seminal contributions and Professor T. M. O'Neil and Dr. J. Danielson for many fruitful discussions.

This work was supported by National Science Foundation Grant No. PHY-0354979.

${ }^{1}$ J. P. Sullivan, S. J. Gilbert, and C. M. Surko, Phys. Rev. Lett. 86, 1494 (2001).

${ }^{2}$ K. H. Knoll, G. Marx, K. Hubner, F. Schweikert, S. Stahl, C. Weber, and G. Werth, Phys. Rev. A 54, 1199 (1996).

${ }^{3}$ T. B. Mitchell, J. J. Bollinger, D. H. E. Dubin, X.-P. Huang, W. M. Itano, and R. H. Baughman, Science 282, 1290 (1998).

${ }^{4}$ N. T. Nakata, G. W. Hart, and B. G. Peterson, in Non-Neutral Plasma Physics IV, edited by F. Anderegg, L. Schweikhard, and C. F. Driscoll
(AIP, New York, 2002), pp. 271-276.

${ }^{5}$ S. A. Prasad and T. M. O’Neil, Phys. Fluids 26, 665 (1983).

${ }^{6}$ A. W. Trivelpiece and R. W. Gould, J. Appl. Phys. 30, 1784 (1959).

${ }^{7}$ D. J. Wineland and H. G. Dehmelt, J. Appl. Phys. 46, 919 (1975).

${ }^{8}$ R. W. Gould, Phys. Plasmas 2, 2151 (1995).

${ }^{9}$ I. Fidone, G. Giruzzi, and G. Taylor, Phys. Plasmas 3, 2331 (1996).

${ }^{10}$ R. L. Stenzel and R. W. Gould, J. Appl. Phys. 42, 4225 (1971).

${ }^{11}$ M. Amoretti, C. Amsler, G. Bonomi et al., Phys. Rev. Lett. 91, 55001 (2003).

${ }^{12}$ N. Meyer-Vernet and C. Perche, J. Geophys. Res. 94, 2405 (1989).

${ }^{13}$ S. Nunomura, D. Sansonov, and J. Goree, Phys. Rev. Lett. 84, 5141 (2000).

${ }^{14}$ A. Gillespie and F. Raab, Phys. Rev. D 52, 577 (1995).

${ }^{15}$ G. Bekefi, Radiation Processes in Plasmas (Wiley, New York, 1966), Sec. 8.4.

${ }^{16}$ J. Evans and M. Loewenthal, Planetary Space Sci. 12, 915 (1964).

${ }^{17}$ F. Anderegg, E. M. Hollmann, and C. F. Driscoll, Phys. Rev. Lett. 81, 4875 (1998).

${ }^{18}$ E. M. Hollmann, F. Anderegg, and C. F. Driscoll, Phys. Plasmas 7, 2776 (2000).

${ }^{19}$ A. Hyatt, Ph.D. thesis, University of California at San Diego, 1988.

${ }^{20}$ D. L. Eggleston, C. F. Driscoll, B. R. Beck, A. W. Hyatt, and J. H. Malmberg, Phys. Fluids B 4, 3432 (1992).

${ }^{21}$ R. C. Davidson, Physics of Non-Neutral Plasmas (Addison-Wesley, New York, 1990), Sec. 5.5.2.

${ }^{22}$ Most of the material of Sec. III has been presented previously in F. Anderegg, N. Shiga, D. H. E. Dubin, and C. F. Driscoll, Phys. Plasmas 10, 1556 (2003). For pedagogical clarity, we briefly describe it in Sec. III.

${ }^{23}$ F. Anderegg, N. Shiga, J. R. Danielson, D. H. E. Dubin, and C. F. Driscoll, Phys. Rev. Lett. 90, 115001 (2003).

${ }^{24}$ D. Fray, American Institute of Physics Handbook (McGraw-Hill, New York, 1963), Sec. 5.

${ }^{25}$ J. Jennings, R. Spencer, and K. C. Hansen, Phys. Plasmas 2, 2630 (1995).

${ }^{26}$ D. Dubin, Phys. Rev. Lett. 66, 2076 (1991).

${ }^{27}$ D. Dubin and T. M. O’Neil, Rev. Mod. Phys. 71, 87 (1999).

${ }^{28}$ M. D. Tinkle, R. G. Greaves, C. Surko, R. Spencer, and G. Mason, Phys. Rev. Lett. 72, 352 (1994)

${ }^{29}$ M. D. Tinkle, R. G. Greaves, and C. Surko, Phys. Plasmas 2, 2880 (1995).

${ }^{30}$ M. Amoretti, G. Bonommi, A. Bouchta et al., Phys. Plasmas 10, 3056 (2003). 